\title{
Review of: "Radiation dose enhancement using gold nanoparticles with a diamond linear accelerator target: a multiple cell type analysis"
}

\author{
Cindy LE LOIREC ${ }^{1}$ \\ 1 Atomic Energy and Alternative Energies Commission
}

Potential competing interests: The author(s) declared that no potential competing interests exist.

The paper deals with the experimental determination of dose enhancement using gold nanoparticules combined with a diamond linear accelerator target. Tests were performed on multiple celle types and also on head-and-nexk cancern celle and finally in zebrafish. Results show an optimization of radiotherapy efficacy which could be of high interest to treat tumors localized near organs at risk.

The objectives, methodology and results of the study are well presented.

The paper can be published but I suggest some minor corrections.

I have indeed some questions related to the medical physics part of the study:

- A True Beam linac from VARIAN was used with a new diamond target embedded in the carroussel. A 2.5 MV beam was chosen with this target. Could you explain this choice of the potential which is nowadays not used in clinical practice, as it is mentionned in the reference paper |6]. This choice could indeed « biased » the results, because the probability of photelectric effect is higher with a $2.5 \mathrm{MV}$ beam than with a $6 \mathrm{MV}$ beam.

Could you explain your choice?

- Physical processes induced by the presence of GNP should be explained with more details: on page 5 it is written « ... due to maximized absorption of characteristic X-rays to consequently optimize Auger emission ». Physical processes are not so simple : photo-electric effect induces emission of photoelectrons (which contributes to the dose enhancement) and also vacancies in the electronic procession of the atom. These vacancies are then filled by other electrons of the procession. This process is accompanied by the emission of X-rays or Auger (or Coster-Kronig) electrons. Actually emission of X-rays induces new vacancies that can produce Auger electrons when they are filled.

Please, describe the physical processes that lead to dose enhancement in more details.

- In the discussion and/or conclusion parts, authors indicate that this « combination therapy could provide an opportunity to escalade the dose to the tumor while minimizing normal tissue complication. » However, before being use in clinics, the phenomenoms that are encountered in this therapy should be well understand and a computation model of the processes should be available, in order to be 
implemented in treatment planification systems. I think that it could be interesting to compare the present results with simulations of electron track structures performed with GEANT4-DNA or other codes able to model ionisation processes at small atomuc level. Such future works should be mentioned in the conclusion because they are of high importance for the clinical applications of such therapies. 